\title{
Determination of Barometric Altimeter Errors for the Orion Exploration Flight Test-1 Entry
}

\author{
Denise L Brown ${ }^{1}$ and Jean-Philippe Munoz ${ }^{2}$ \\ Odyssey Space Research, LLC, Houston TX 77058 \\ Robert $\mathrm{Gay}^{3}$ \\ NASA-Johnson Space Center, Houston, TX 77058
}

The EFT-1 mission is the unmanned flight test for the upcoming Multi-Purpose Crew Vehicle (MPCV). During entry, the EFT-1 vehicle will trigger several Landing and Recovery System (LRS) events, such as parachute deployment, based on onboard altitude information. The primary altitude source is the filtered navigation solution updated with GPS measurement data. The vehicle also has three barometric altimeters that will be used to measure atmospheric pressure during entry. In the event that GPS data is not available during entry, the altitude derived from the barometric altimeter pressure will be used to trigger chute deployment for the drogues and main parachutes. Therefore it is important to understand the impact of error sources on the pressure measured by the barometric altimeters and on the altitude derived from that pressure.

There are four primary error sources impacting the sensed pressure: sensor errors, Analog to Digital conversion errors, aerodynamic errors, and atmosphere modeling errors. This last error source is induced by the conversion from pressure to altitude in the vehicle flight software, which requires an atmosphere model such as the US Standard 1976 Atmosphere model. There are several secondary error sources as well, such as waves, tides, and latencies in data transmission.

Typically, for error budget calculations it is assumed that all error sources are independent, normally distributed variables. Thus, the initial approach to developing the EFT-1 barometric altimeter altitude error budget was to create an itemized error budget under these assumptions. This budget was to be verified by simulation using high fidelity models of the vehicle hardware and software.

The simulation barometric altimeter model includes hardware error sources and a datadriven model of the aerodynamic errors expected to impact the pressure in the midbay compartment in which the sensors are located. The aerodynamic model includes the pressure difference between the midbay compartment and the free stream pressure as a function of altitude, oscillations in sensed pressure due to wake effects, and an acoustics model capturing fluctuations in pressure due to motion of the passive vents separating the barometric altimeters from the outside of the vehicle.

The CM compartment pressure model used the standard set of EFT-1 PTR-3 dispersed trajectories from Osiris, the CAP aerodynamic loads database, and variable discharge coefficients generated using Computational Fluid Dynamics (CFD) to assess the altitude-

\footnotetext{
${ }^{1}$ Orion Barometric Altimeter Flight Software Lead, 1120 NASA Parkway, Houston, TX 77058.

${ }^{2}$ Engineer Specialist, 1120 NASA Parkway, Houston, TX 77058.

${ }^{3}$ Orion Absolute Navigation Lead - NASA, 2101 NASA Parkway, Houston, TX 77058.
} 
dependent pressure inside midbay compartment $F$, where the barometric altimeters are located. Conservative assumptions were made on model uncertainties. The wake pressure model was derived from results of the 05-CA wind tunnel test. The results were spotchecked by CFD/LES simulation of the crew module (CM) in free-stream. The acoustics model was provided by Loads \& Dynamics using entry aerodynamic environments adjusted for CM compartment effects. These models have two key limitations: CM RCS jet interaction was neglected and the supersonic data for the CM wake pressure model was sparse.

Monte Carlo analysis was performed to assess each of the four main barometric altimeter altitude error sources independently. For these analyses, only one of the four error sources was enabled in order to assess that source as a stand-alone, independent source. Sensor and A/D conversion error characteristics were taken from hardware specifications and performance requirements. The Monte Carlo results therefore matched expected behavior for these error sources. The aerodynamic errors were modeled as described above, and bounding aerodynamic errors were calculated based on the Monte Carlo analysis results. Atmosphere modeling errors were also assessed via Monte Carlo simulation. The Osiris simulation uses GRAM2007 to model the earth's atmosphere; the difference between 'truth' altitude based on GRAM2007 and the altitude output by the barometric altimeter flight software was calculated and the errors quantified. An itemized error budget was then calculated using these results and the expected errors from the minor error sources.

At altitudes above 50,000 feet, the aerodynamic errors are the dominant error source by an order of magnitude. Until 8000 feet, these are still the largest error source, but the $A / D$ conversion errors are of the same magnitude. At altitudes below 15,000 feet the A/D conversion errors are the largest error source.

The itemized error budget results were compared to results from Monte Carlo simulation with all four of the main altitude error sources enabled simultaneously. The simulation results did not match the itemized error budget; the errors at higher altitudes in the simulation were much larger than those predicted by the itemized error budget, while errors at low altitudes in the simulation were slightly smaller than those predicted by the itemized error budget.

Needless to say, this prompted an in-depth investigation to ascertain why the itemized error budget and the simulated results differed so drastically at altitudes above 35,000 feet. The simulation model outputs were verified by Subject Matter Experts (SMEs) to rule out simulation errors. Eventually, the differences between the itemized budget results and the Monte Carlo simulation results was traced to a violation of one of the assumptions used to create the itemized error budget. The atmosphere modeling errors are not independent of the other error sources.

All of the main error sources other than the atmosphere modeling errors cause the sensed pressure to deviate from the 'truth' atmospheric pressure, where truth pressure is defined as pressure from the GRAM2007 atmosphere model. However, the atmosphere modeling error is an artifact of the conversion from sensed pressure to altitude. In the flight software, a 1976 US Standard Atmosphere model is used to convert pressure to altitude. Errors in sensed pressure result in larger atmosphere modeling errors than those predicted solely by differences between GRAM2007 and the 1976 US Standard Atmosphere model. Thus the atmosphere modeling errors in the simulation with all error sources enabled 
differed from the atmosphere modeling error with all other error sources disabled. At higher altitudes, aerodynamic errors cause large deviations between sensed pressure and free stream atmospheric pressure. This is why the itemized error budget values differed so significantly at higher altitudes. At lower altitudes, the error in sensed pressure is much smaller because aerodynamic effects were much smaller, so the atmosphere modeling error is also much smaller and the itemized error budget results more closely matched the full Monte Carlo analysis results.

The correlation between the sensed pressure error and the atmosphere modeling errors violates the independence of error sources assumed by the itemized error budget method. It should also be noted that the distributions of the individual error sources was not taken into account in the itemized error budget. The itemized error budget approach assumes normally distributed variables; at higher altitudes, neither the atmosphere modeling errors nor the aerodynamic errors appear to be normally distributed.

In cases where the distribution of the individual error sources is unknown and the error sources are correlated, itemized error budget approaches that assume independent normally distributed variables cannot be applied with any validity. Thus full simulation with highfidelity error modeling that captures interaction between error sources and does not make assumptions about the distribution of each error source is the only way to adequately characterize the total errors.

The results of the error budget analysis will be used to set trigger altitudes for EFT-1 LRS events when using the barometric altimeter altitude. These trigger altitudes will take the expected errors into account, which will enable the vehicle to deploy the drogue and main chutes within the correct altitude ranges even with sensor errors. 